\title{
O Efeito de Instruções e de Mudanças de Instruções sobre o Comportamento em DRL
}

\author{
Carlos Eduardo Costa ${ }^{1}$ \\ Departamento de Psicologia Geral e Análise do Comportamento da Universidade Estadual \\ de Londrina, Londrina, PR, Brasil \\ Fernanda Castanho Calixto \\ Universidade Federal de São Carlos, São Carlos, SP, Brasil \\ Roberto Alves Banaco \\ Paradigma Centro de Ciência e Tecnologia do Comportamento, São Paulo, SP, Brasil
}

\begin{abstract}
Resumo
O objetivo foi investigar o efeito da história comportamental com regras correspondentes, discrepantes e mínimas sobre o seguimento de uma regra discrepante subsequente. Participaram 20 estudantes universitários distribuídos em quatro grupos. Nas duas fases do estudo a tarefa foi clicar um botão e ganhar pontos, liberados de acordo com um DRL 5 segundos. Na Fase 1, os grupos foram caracterizados de acordo com a instrução fornecida: Grupo Controle e Grupo IM (Instrução Mínima), Grupo IC (Instrução Correspondente) e Grupo ID (Instrução Discrepante). Na Fase 2 o Grupo Controle recebeu novamente a instrução mínima e os demais grupos receberam uma instrução discrepante. A IM informava que os participantes deveriam ganhar pontos usando o mouse; a IC dizia que deveriam pressionar o botão a cada $5 \mathrm{~s}$ e a ID dizia que deveriam pressionar o botão a cada segundo. Os resultados do presente estudo sugeriram que as histórias com instruções correspondentes (Grupo IC) e discrepantes (Grupo ID) na Fase 1 aumentaram o tempo necessário para o contato com as contingências programadas na Fase 2 e a história com instruções mínimas aumentou a probabilidade de sensibilidade comportamental.
\end{abstract}

Palavras-chave: História comportamental, comportamento governado por regras, resistência à mudança.

\section{Effect of Rules and Rule Change on the Behavior in DRL}

\begin{abstract}
This study aimed to investigate the effect of behavioral history with correspondent, discrepant and minimum rules on the following of a subsequent discrepant rule. The participants were twenty students distributed into four groups. Along two phases the tasks was clicking a button and earn points released in accordance with a DRL 5 seconds. In Phase 1, groups were assigned according to the instruction provided to them: Group Control and Group IM (Minimum Instruction), Group IC (Correspondent Instruction) and Group ID (Discrepant Instruction). In Phase 2, participants of Group Control received the same
\end{abstract}

Endereço para correspondência: Universidade Estadual de Londrina, Centro de Ciências Biológicas, Departamento de Psicologia Geral e Análise do Comportamento, Rodovia Celso Garcia Cid, Km 380, Caixa Postal 10.011, Londrina, PR, Brasil, 86057-970. Fone: (43) 3371-4227. E-mail: caecosta@uel.br

Fernanda C. Calixto era discente do Mestrado em Análise do comportamento da Universidade Estadual de Londrina (UEL) e bolsista pela Fundação Araucária durante a realização desse trabalho. Os autores agradecem a ajuda da discente de graduação em Psicologia da UEL, Angela Cândida da Costa, pela coleta e tabulação parcial dos dados da presente pesquisa. 
minimum instruction and participants from the other groups received a new discrepant instruction. The MI informed that participants should earn points using the mouse; The CI informed that participant should press the button every 5 seconds and the DI informed that they should press the button every second. Results obtained suggested that histories with correspondent (Group IC) and discrepant (Group ID) instructions increased the time needed to contact the programmed contingencies in Phase 2 and the history with minimal instructions increased the likelihood of behavioral sensitivity.

Keywords: Behavioral history, rules governed behavior, resistance to change.

\section{El efecto de la Instrucciones y de Cambios de Instrucciones sobre el Comportamiento en DRL}

\section{Resumen}

El objetivo fue investigar el efecto de la historia comportamental con reglas correspondientes, discrepantes y mínimas sobre el seguimiento de una regla discrepante subsecuente. Participaron 20 universitarios distribuidos en cuatro grupos. En las dos fases del estudio la tarea fue clicar en un botón y ganar puntos, liberados de acuerdo con un DRL 5 segundos. En la Fase 1, los grupos fueron caracterizados de acuerdo con la instrucción suministrada: Grupo Control y Grupo IM (instrucción mínima), Grupo IC (Instrucción Correspondiente) y Grupo ID (Instrucción Discrepante). En la Fase 2 el Grupo Control recibió nuevamente la instrucción mínima y los demás grupos recibieron una instrucción discrepante. La IM informaba que los participantes deberían ganar puntos usando el ratón; la IC decía que deberían presionar el botón a cada $5 \mathrm{~s}$ y la ID decía que deberían presionar el botón a cada segundo. Los resultados del presente estudio sugirieron que las historias con instrucciones correspondientes (Grupo IC) y discrepantes (Grupo ID) en la Fase 1 aumentaron el tiempo necesario para el contacto con las contingencias programadas en la Fase 2 y la historia con instrucciones mínimas aumentó la posibilidad de sensibilidad comportamental.

Palabras clave: Historia comportamental, comportamiento gobernado por reglas, resistencia al cambio.

Descrições verbais de relações funcionais entre a emissão de comportamentos e seu(s) produto(s) no ambiente facilitam a aprendizagem de novos repertórios por não requerer, necessariamente, o contato direto com as contingências. Neste caso, a relação entre eventos ambientais e as consequências que os seguem é especificada por um enunciado denominado de regra ou instrução (Albuquerque \& Paracampo, 2010; Matos, 2001; Skinner 1969). Cerutti (1989) aponta que os termos "regras" e "instruções", normalmente, são termos de uso equivalentes, pois ambos se referem a descrições verbais das contingências. No presente trabalho os termos "regra" e "instrução" serão utilizados como sinônimos e o comportamento sob o controle de regras/ instruções será denominado de comportamento controlado ou governado por regras. Um com- portamento é dito controlado por regras quando ocorre de acordo com uma descrição verbal das contingências e sem o contato prévio com as consequências imediatas que produz. Caso o comportamento se mantenha após o contato com suas consequências imediatas, ele passa a ser considerado como controlado pela interação da regra com as consequências (Albuquerque, dos Reis, \& Paracampo, 2008; Matos, 2001).

Apesar de instruções facilitarem a aquisição de comportamentos, se comportar sob o controle de regras pode ser desvantajoso quando ocorrem mudanças não sinalizadas nas contingências (e.g., Albuquerque \& Paracampo, 2010; AthaydeNeto, Costa, \& Banaco, 2015; Calixto, Ponce, \& Costa, 2014; Skinner, 1969). O comportamento estabelecido por regras que se mantem a despeito das consequências imediatas é denominado de 
"insensível às contingências". (Albuquerque \& Paracampo, 2010; Catania, 1998/1999; Hayes, Brownstein, Haas, \& Greenway, 1986; Lowe, 1979; Madden, Chase, \& Joyce, 1998; Shimoff, Catania, \& Matthews, 1981). A insensibilidade de um comportamento às contingências pode ser investigada, pelo menos, de duas formas: (a) a mesma regra é mantida inalterada e ocorrem mudanças nas contingências programadas ou (b) diferentes regras são fornecidas e a contingência em vigor é mantida constante (cf. Albuquerque et al., 2008, sobre as duas maneiras de se estudar a sensibilidade do comportamento).

Diversas pesquisas (e.g., Albuquerque, Silva, \& Paracampo, 2014; Athayde-Neto et al., 2015; Calixto et al., 2014; Catania, Matthews, \& Shimoff, 1982; Galizio, 1979; Paracampo \& Albuquerque, 2004) investigaram variáveis que afetam a manutenção do comportamento controlado por instruções perante alterações nas contingências programadas.

A história experimental com instruções correspondentes e discrepantes tem sido uma das variáveis investigadas no estudo da sensibilidade comportamental. Instruções são chamadas de "correspondentes" quando se comportar de acordo com elas garante a obtenção dos reforços programados e são chamadas de "discrepantes" quando se comportar de acordo com elas não garante a obtenção dos reforços programados (Albuquerque et al., 2014).

Albuquerque, de Souza, Matos, e Paracampo (2003, Experimento 1), por exemplo, investigaram o efeito da exposição prévia a uma instrução correspondente às contingências de reforço sobre o seguimento subsequente de uma instrução discrepante. Oito estudantes universitários foram expostos a um procedimento de escolha de acordo com o modelo. Em cada tentativa um estímulo modelo e três de comparação eram apresentados. O participante deveria apontar para os estímulos de comparação em uma sequência predeterminada pelo experimentador. Cada estímulo de comparação possuía apenas uma dimensão - cor (C), espessura (E) ou forma (F) - em comum com o modelo e diferia nas demais. Os participantes foram distribuídos em duas condições: Correspondente-Discrepante
(C-D) ou Discrepante-Correspondente-Discrepante (D-C-D). Na Fase 1, para os participantes de ambas as condições, não era fornecida nenhuma instrução que especificasse a sequência de apontar e nenhuma resposta era reforçada. Para os participantes da Condição C-D as Fases 2 e 3 eram iniciadas com instruções correspondentes e discrepantes, respectivamente. As sequências de respostas instruídas eram CEF ou EFC na condição correspondente (e estas eram as sequências reforçadas) e FCE ou CFE na condição discrepante (e CEF e EFC continuavam a ser as sequências reforçadas). Para os participantes da Condição D-C-D as Fases 2, 3 e 4 eram iniciadas com instruções discrepantes, correspondentes e discrepantes, respectivamente. Para os participantes de ambas as condições, a escolha dos estímulos de comparação na sequência especificada pela instrução correspondente era reforçada (acréscimo de pontos em um contador) em Razão Fixa (FR) 4, enquanto a escolha dos estímulos de comparação em qualquer outra sequência não era reforçada. Os resultados indicaram que todos os participantes, de ambas as condições, seguiram as instruções fornecidas, correspondentes ou discrepantes, independentemente do ganho de pontos e da ordem em que as instruções foram fornecidas. Os resultados sugeriram que o contato com a discrepância entre instrução e contingência (i.e., a não obtenção de pontos por seguir a instrução discrepante) não foi suficiente para o abandono de instruções discrepantes. A história experimental, bem sucedida, de seguir a instrução correspondente (Condição C-D) pode ter contribuído para o seguimento subsequente da instrução discrepante. Todavia, os participantes da Condição D-C-D seguiram a instrução discrepante mesmo na primeira exposição a elas, o que indica que, embora a história experimental de seguir instruções correspondentes possa contribuir para o seguimento de instruções discrepantes, o seguimento de instruções discrepantes não é uma função exclusiva da história experimental de seguir instruções correspondentes.

Os autores levantaram a hipótese de que o contato com a instrução correspondente, sem a garantia de contato prévio com as contingências programadas por exposição direta, contribuiu 
para o seguimento da instrução discrepante. Por esse motivo, Albuquerque et al. (2003, Experimento 2) conduziram um segundo experimento no qual o contato direto com as contingências (garantido por um critério de obtenção de um número mínimo de pontos para a mudança de fase) era pré-requisito para o posterior fornecimento de instruções correspondentes e discrepantes. Participaram oito estudantes universitários que passaram por quatro fases: instrução mínima, instrução discrepante, instrução correspondente, instrução discrepante. A tarefa experimental foi idêntica ao Experimento 1. Durante a Fase 1 nenhuma instrução sobre a sequência de respostas era fornecida e a sequência CEF foi reforçada em FR 1, aumentando gradualmente para FR 4 e a fase era encerrada quando o participante obtinha 20 pontos em FR 4, sendo os quatro últimos pontos consecutivos. Caso o participante não obtivesse os 20 pontos na Fase 1 sua participação no experimento era encerrada. Na Fase 2 a instrução indicava a sequência FCE e pontos eram liberados para a sequência CEF (Instrução Discrepante). Na Fase 3, a instrução indicava a sequência $\mathrm{EFC}$ e os pontos eram liberados para a sequência EFC e CEF (Instrução Correspondente). Finalmente, na Fase 4, a instrução indicava a sequência FCE, mas pontos eram liberados para a sequência CEF (Instrução Discrepante). Dos seis participantes, que atingiram o critério de acertos na primeira fase, quatro seguiram as instruções (correspondente e discrepante) ao longo do experimento, sugerindo que o contato prévio com as contingências (com instrução mínima) não foi suficiente para garantir o abandono da instrução discrepante subsequente. Dois participantes se comportaram de acordo com as contingências programadas, deixando de seguir as instruções discrepantes.

Albuquerque e Silva (2006) realizaram um estudo para investigar como a história de aquisição de um comportamento alternativo pode afetar o seguimento subsequente de instruções discrepantes.Maisespecificamente, oexperimento manipulou a forma como o comportamento alternativo à instrução foi estabelecido (por contingência, por instruções correspondentes ao comportamento verbal ou por instruções correspondentes ao comportamento não verbal) e se esse comportamento alternativo mudaria com a alteração nas contingências de reforço, antes da introdução da instrução discrepante. $\mathrm{O}$ procedimento foi de escolha de acordo com o modelo semelhante ao de Albuquerque et al. (2003). Participaram nove estudantes universitários que deveriam emitir respostas não verbais (apontar os estímulos de comparação em sequência) e respostas verbais (descrever a ordem em que os estímulos de comparação deveriam ser apontados). Os participantes foram distribuídos em três condições que se diferenciavam pela instrução fornecida no início da primeira sessão. $\mathrm{Na}$ Condição 1 era fornecida aos participantes uma instrução mínima; na Condição 2 era fornecida a instrução correspondente à resposta não-verbal e na Condição 3 era fornecida uma instrução correspondente à resposta verbal. $\mathrm{Na}$ Sessão 1 apontar os estímulos de comparação na sequência CEF e FCE (resposta não-verbal) e descrever a mesma sequência (resposta verbal) era seguido pela obtenção de três pontos. $\mathrm{Na}$ Sessão 2, sem sinalização, a sequência reforçada passou a ser ECF e CFE para a resposta não verbal e verbal. A Sessão 3 foi idêntica à Sessão 1 e no início da Sessão 4 os participantes das três condições foram expostos à instrução discrepante (descrevia que as sequências corretas eram FEC e ECE), mas as contingências eram idênticas àquelas das Sessões 1 e 3.

Os resultados indicaram que, independente da instrução fornecida na primeira sessão, os participantes que alteraram seu desempenho de acordo com as mudanças nas contingências programadas na Sessão 2 (i.e., o comportamento mostrou-se sensível a alteração nas contingências), não se comportaram de acordo com a instrução discrepante (Sessão 4). Este resultado sugere que o controle prévio pela contingência, e não a forma como o comportamento alternativo foi adquirido, diminuiu a probabilidade de controle instrucional quando instruções discrepantes foram fornecidas subsequentemente (Albuquerque \& Silva, 2006).

Albuquerque et al. (2014) realizaram um estudo com 10 estudantes universitários semelhante ao descrito acima. A Sessão 1, com instruções 
mínimas, nenhuma sequencia era reforçada. $\mathrm{Na}$ Sessão 2, para os participantes do Grupo $1(n=5)$ a sequência correta de apontar os estímulos de comparação foi estabelecida por reforço diferencial e para os participantes do Grupo $2(n=5)$ foi estabelecida por uma instrução correspondente. Na transição da Sessão 2 para a 3 ocorria uma mudança nas contingências de reforço e na transição da Sessão 3 para a 4 ocorria a introdução de uma instrução discrepante. Assim como no estudo de Albuquerque e Silva (2006), os resultados demonstraram que os três dos quatro participantes que alteraram o desempenho de acordo com as mudanças nas contingências na Sessão 3 não seguiram a instrução discrepante na Sessão 4 (dois participantes do Grupo 1 e um do Grupo 2) e os participantes que não alteraram o comportamento com a alteração das contingências na Sessão 2 seguiram a instrução discrepante na Sessão 4 (um participante do Grupo 1 e quatro do Grupo 2). Um participante do Grupo 1 não completou a Sessão 2 e foi excluído do restante do procedimento.

Nos estudos descritos previamente (Albuquerque et al., 2003; Albuquerque \& Silva, 2006 e Albuquerque et al., 2014), em ao menos uma fase, os participantes entraram em contato com instruções correspondentes com as contingências programadas. Uma interpretação possível, dos resultados dos participantes que seguiram a instrução discrepante é que a história experimental de reforço de se comportar de acordo uma instrução correspondente pode ter sido suficiente para manter o seguimento das instruções discrepantes subsequente (mas ver Albuquerque et al., 2003 sobre a possibilidade de a história experimental com regras correspondentes não ser a única variável relevante). Outros estudos (e.g., Calixto et al., 2014; Martinez \& Tamayo, 2005) investigaram o efeito de histórias experimentais com instruções discrepantes e com instruções correspondentes, isoladamente, sobre a sensibilidade do seguimento de regra às contingências programadas.

Martinez e Tamayo (2005, Experimento 2), por exemplo, investigaram o efeito da história experimental com instruções correspondentes e discrepantes sobre o comportamento de seguir instruções subsequentes. O procedimento utilizado foi o de escolha de acordo com o modelo no qual a tarefa consistia em escolher o estímulo de comparação que era diferente, idêntico ou similar (na forma ou na cor) ao estímulo modelo. Os participantes eram distribuídos randomicamente em quatro grupos com diferentes histórias experimentais. Em todos os grupos as respostas corretas durante as primeiras três fases de treino (12 sessões) consistiam em escolher o estímulo de comparação diferente (em cor e forma) do modelo e as instruções, fossem correspondentes ou discrepantes, eram mantidas constantes. $\mathrm{Na}$ quarta fase as instruções ou as contingências poderiam mudar. Para os participantes do Grupo 1 a instrução era correspondente em todas as fases do estudo; para os do Grupo 2 a instrução era discrepante em todas as fases do estudo; para os participantes do Grupo 3 a instrução era correspondente nas três primeiras fases e discrepante na quarta fase e para os do Grupo 4 a instrução discrepante era apresentada nas três primeiras fases do estudo e a instrução correspondente era fornecida na quarta fase.

Os resultados indicaram que a maioria dos participantes expostos à instrução discrepante nas três primeiras fases do estudo (nove de 10 participantes dos Grupos 2 e 4 ) abandonou o seguimento da instrução discrepante ao longo das três fases e, na Fase 4, os participantes do Grupo 2 não seguiram a nova instrução discrepante e os do Grupo 4 seguiram a instrução correspondente. Os participantes para os quais foram fornecidas instrução correspondente nas três fases iniciais (Grupos 1 e 3) seguiram a instrução correspondente e, na Fase 4, as instruções foram seguidas, independentemente de elas serem correspondentes (Grupo 1) ou discrepantes (Grupo 3). Esses resultados, sugerem que história de exposição às instruções correspondentes, aumenta a probabilidade de que, em fase subsequente, os participantes sigam uma instrução discrepante, enquanto que, a história prévia com instruções discrepantes facilitou o abandono do controle instrucional quando uma nova instrução discrepante foi apresentada (Martinez \& Tamayo, 2005).

Calixto et al. (2014, Experimento 2) investigaram o efeito da exposição prévia a uma de 
diferentes instruções sobre a sensibilidade do comportamento ao seguimento de uma instrução discrepante quando ocorre mudança nas contingências. Quinze estudantes universitários foram distribuídos em três grupos $(n=5)$ que se diferenciavam pela instrução recebida no início do experimento. A tarefa consistia em pressionar o botão do mouse com o cursor sobre um retângulo (botão de respostas) que aparecia na posição central inferior do monitor. Na Fase 1 todos os participantes foram expostos a um programa de reforço-diferencial-de-baixas-taxas (DRL) $5 \mathrm{~s}$. Os participantes do Grupo IM foram expostos a uma instrução mínima (informava que o participante deveria ganhar pontos usando o mouse), os do Grupo IC foram expostos a uma instrução correspondente ao DRL (pressionar o botão de 5 em 5 segundos) e os do Grupo 3 a uma instrução discrepante ao DRL (pressionar o botão pelo menos uma vez por segundo). Na Fase 2, todos os participantes foram expostos à Extinção, sem qualquer instrução ou sinalização de mudança nas contingências. Na Fase 1, apesar de quatro participantes do Grupo ID e de dois do Grupo IM terem emitido altas taxas de respostas iniciais, todos os participantes (dos três grupos) finalizaram a fase emitindo baixas taxas de respostas. Na Fase 2, quando a Extinção estava em vigor, três de quatro participantes do Grupo IC obtiveram proporção de mudança da taxa de respostas menor que $50 \%$ (i.e., foram menos sensíveis à alteração na contingência) enquanto que três de cinco participantes de cada um dos demais grupos mudaram a taxa de respostas em mais de $50 \%$ (i.e., foram mais sensíveis à alteração nas contingências). Esses resultados sugerem que, em contingências nas quais seguir uma instrução discrepante (Grupo ID) inviabiliza o ganho de reforços programados e os pontos podem ser obtidos emitindo taxas de respostas diferentes daquelas descritas na instrução, como ocorreu no DRL, o abandono do controle instrucional é mais provável e que a história prévia com a instrução correspondente (Grupo IC) pode afetar a resistência do comportamento à mudança quando os reforçadores são suspensos.

Os resultados de Calixto et al. (2014) e os de Martinez e Tamayo (2005) sugeriram que a história prévia com instruções correspondentes aumenta (a) a probabilidade do seguimento de instrução discrepante (Martinez \& Tamayo, 2005) e a resistência do comportamento à mudança (Calixto et al., 2014) e (b) o contato prévio com instruções discrepantes diminui a probabilidade do seguimento de instruções discrepantes subsequentes (Calixto et al., 2014).

Os estudos citados previamente investigaram a sensibilidade do comportamento de diferentes maneiras. No estudo de Albuquerque et al. (2003), por exemplo, regras diferentes foram fornecidas e as contingências programadas eram mantidas constantes, enquanto que no estudo de Calixto et al. (2014) e parte do procedimento de Martinez e Tamayo (2005), o procedimento mais tradicional de manter a regra e alterar a contingência foi adotado. A tarefa experimental também diferiu entre os estudos (i.e., escolha de acordo com o modelo em um procedimento de teste discreto $v s$. pressionar um botão em um modelo de operante livre). O experimento de $\mathrm{Ca}$ lixto et al. poderia ser replicado usando o procedimento menos tradicional (como aqueles utilizados por Albuquerque e seus colaboradores) de manter as contingência de reforço e alterar a instrução. Essa mudança poderia lançar luz sobre a generalidade dos resultados com procedimentos diferentes.

O presente estudo teve o objetivo de investigar o efeito da história experimental com instruções correspondentes, discrepantes ou mínimas (i.e., reforço diferencial) sobre o seguimento de uma instrução discrepante subsequente. No estudo de Calixto et al. (2014) as instruções fornecidas se mantiveram constantes ("pressione o botão rapidamente") ao mesmo tempo em que houve alteração no programa de reforço (de DRL para Extinção). No presente estudo o programa de reforço foi mantido constante (DRL nas duas fases) e a instrução foi modificada (cf. Albuquerque et al., 2008, sobre as duas maneiras de se estudar a sensibilidade do comportamento).

Além de avaliar a generalidade dos resultados de Calixto et al. (2014) utilizando um procedimento alternativo para se avaliar o controle instrucional, manter o DRL na Fase 2 permitiu avaliar se o eventual contato com as contingên- 
cias na Fase 1 (i.e., obter reforços por responder após uma pausa de, pelo menos, $t$ segundos) aumentou a probabilidade de abandono da instrução discrepante na Fase 2 quando reforços estavam disponíveis para o mesmo comportamento emitido na Fase 1 (em vez de suspender o reforço como em Calixto et al., 2014).

\section{Método}

\section{Participantes}

Participaram 21 estudantes universitários (exceto do curso de Psicologia), de ambos os sexos, com idades entre 18 e 32 anos sem queixa ou diagnóstico de LER (Lesão por Esforço Repetitivo) ou DORT (Distúrbio Osteomuscular Relacionado ao Trabalho). O participante P20 não quis concluir a pesquisa e, por isso, foram analisados os resultados de 20 participantes.

\section{Equipamentos, Instrumento e Local}

A coleta de dados ocorreu em duas salas experimentais de aproximadamente $3 \mathrm{~m}^{2}$ cada. Cada sala continha um computador do tipo PC, com monitor em cores de 14 polegadas, mouse e teclado padrões; uma mesa; uma cadeira; um ventilador; um fone de ouvido e uma filmadora com tripé. O software utilizado na coleta de dados foi o ProgRef v3.1. (Costa \& Banaco, 2002, 2003).

\section{Procedimento}

Antes da sessão experimental, os participantes liam o Termo de Consentimento Livre e Esclarecido (TCLE) que, em linhas gerais, informava que o objetivo da pesquisa era "estudar algumas variáveis que possam afetar o modo como as pessoas se comportam em determinadas situações"; que seriam realizadas duas sessões com duração de 20 minutos cada; que a tarefa experimental seria "acumular o maior valor monetário possível (que apareceria na tela do computador)"; que o valor acumulado seria recebido em dinheiro ao final de cada sessão; que o participante usaria um fone de ouvido durante a sessão do qual soaria um "ruído branco ("chiado')" para isolamento acústico e que poderia abandonar a pesquisa, a qualquer momento, sem prejuízos.

Os participantes foram distribuídos em quatro grupos que se diferenciaram pelo tipo de instrução recebida. A Tabela 1 resume o procedimento experimental.

Tabela 1

Instruções Fornecidas e Programa de Reforço em Vigor na Fase 1 e na Fase 2 para os Participantes dos Quatro Grupos

\begin{tabular}{cccc}
\hline Grupos $^{\mathrm{a}}$ & Tipo de Instrução $^{\mathrm{b}}$ & Fase 1 & Fase 2 \\
\hline Controle & IM-IM & DRL 5 s & DRL 5 s \\
IM & IM-ID & DRL 5 s & DRL 5 s \\
IC & IC-ID & DRL 5 s & DRL 5 s \\
ID & ID-ID & DRL 5 s & DRL 5 s \\
\hline
\end{tabular}

Nota. IM=Instrução Mínima; ID=Instrução Discrepante; IC=Instrução Correspondente.

${ }^{\mathrm{a}} n=5 ;{ }^{\mathrm{b}}$ Instrução Fase 1-Fase 2

Cada participante era instruído a deixar todo material, incluindo celular e relógio, fora da sala experimental e só então era convidado a entrar na sala, sentar-se e ler as seguintes instruções (denominadas "Instruções Mínimas", IM), fornecidas a todos os participantes, independentemente do grupo ao qual pertenciam.
Seu objetivo será ganhar o maior valor em dinheiro que puder utilizando apenas o mouse. O valor ganho em dinheiro aparecerá em uma janela, que se localizará na parte superior da tela do computador na posição central. Com 20 minutos de sessão o procedimento será interrompido, o 
experimentador pagará o valor ganho e uma nova sessão de 20 minutos será realizada. O experimentador não está autorizado a dar qualquer informação adicional. Caso haja dúvidas, releia o texto acima e prossiga $o$ experimento. Bom trabalho!

Adicionalmente a estas instruções, os participantes receberam as instruções descritas abaixo, antes de cada fase experimental, de acordo com o grupo a que pertenciam (ver Tabela 1):

Instrução Correspondente (IC): "Para ganhar pontos você deve pressionar o botão do mouse de 5 em 5 segundos".

Instrução Discrepante (ID): "Para ganhar pontos você deve pressionar o botão do mouse pelo menos uma vez por segundo".

Após a leitura das instruções os participantes colocavam o fone de ouvido, a filmadora era ligada e a sessão experimental era iniciada assim que o botão "Iniciar", presente no monitor, fosse pressionado. $\mathrm{O}$ procedimento era realizado em duas sessões de 20 minutos cada com intervalo de, aproximadamente, 3 minutos entre elas.

Durante a sessão experimental o monitor do computador exibia um fundo cinza, com dois botões e um contador. Um botão retangular no centro inferior (botão de respostas) e um botão retangular no canto superior direito do monitor, denominado botão de resposta de consumação (BRC). Era considerada uma resposta cada clique no botão esquerdo do mouse quando o ponteiro estivesse sobre o botão de respostas. Quando cumprida a exigência do programa de reforço (DRL $5 \mathrm{~s}$, descrito mais adiante), aparecia um smile (figura estilizada de uma carinha risonha) no canto superior direito do monitor, abaixo do BRC (sobre a função da resposta de consumação ver Costa, Patsko, \& Becker, 2007). Um clique sobre o BRC, enquanto o smile estivesse presente, produzia o desaparecimento do smile e o acúmulo do valor em dinheiro ( $\mathrm{R} \$ 0,03)$ em um contador no centro da tela, acima do botão de respostas.

Fase 1. Todos os participantes foram expostos a um DRL $5 \mathrm{~s}$. Neste programa de reforço um smile aparecia no monitor para cada resposta que fosse precedida por uma pausa de pelo menos 5 segundos, contada desde o início da sessão ou do aparecimento do smile anterior. Respostas emitidas antes desse intervalo de tempo zeravam o cronômetro e iniciavam um novo intervalo. $\mathrm{O}$ tempo gasto para migrar o cursor do mouse do botão de respostas ao BRC, clicá-lo e retornar o cursor do mouse ao botão de respostas estava contido no intervalo do DRL. Respostas emitidas no botão de respostas após o aparecimento do smile e antes da resposta de consumação eram registradas e zeravam o intervalo de tempo do DRL. Todavia, se um smile estivesse no monitor, outro smile não aparecia sem que o participante clicasse no BRC (i.e., não havia "acúmulo" de smiles). Após o término desta fase, o participante saía da sala experimental, o valor ganho pelo participante era pago em dinheiro, a Fase 2 era programada e o participante voltava para a sala experimental.

Fase 2. A mesma instrução mínima era fornecida aos participantes do Grupo Controle enquanto os participantes dos Grupos IM, IC e ID receberam instruções discrepantes ("Para ganhar pontos você deve pressionar o botão do mouse pelo menos uma vez por segundo"). O DRL $5 \mathrm{~s}$ estava em vigor tal como na Fase 1.

\section{Resultados}

A Figura 1 exibe a frequência de respostas por minuto de cada participante durante as duas fases do experimento. Os quatro grupos, diferenciados pela instrução recebida na primeira fase do experimento, estão representados pelas colunas verticais, sendo que a primeira coluna corresponde ao Grupo Controle, a segunda ao Grupo IM, a terceira ao Grupo IC e a última coluna corresponde ao Grupo ID. Para facilitar a visualização dos dados foram construídas três escalas para o eixo $y(50,200$ e $500 \mathrm{R} / \mathrm{min})$, exibidos em ordem decrescente em cada coluna da Figura 1. A mudança da Fase 1 para a Fase 2 foi marcada por uma linha tracejada vertical no centro de cada gráfico. Os números, que aparecem em cada fase do estudo, representam a porcentagem de reforços obtida pelo participante em comparação à quantidade de reforço possível de ser obtido.

A Tabela 2 exibe a média da taxa de respostas e a porcentagem de reforços obtidos dos 5 minutos iniciais (Min INI) e dos 5 minutos 
finais (Min FIM) de cada fase do experimento e a proporção de mudança (Prop Mud). A proporção de mudança foi calculada dividindo a taxa de respostas dos 5 minutos finais da Fase 2 pela taxa de respostas dos 5 minutos finais da Fase 1 (cf. Calixto et al., 2014; Nevin, 1974).
Valores próximos de 1 indicam menor proporção de mudança. Primeiramente serão descritos os resultados da Fase 1, apontando resultados exibidos tanto na Figura 1 quanto na Tabela 2 e, em seguida, serão descritos os resultados da Fase 2 dos participantes de cada grupo.

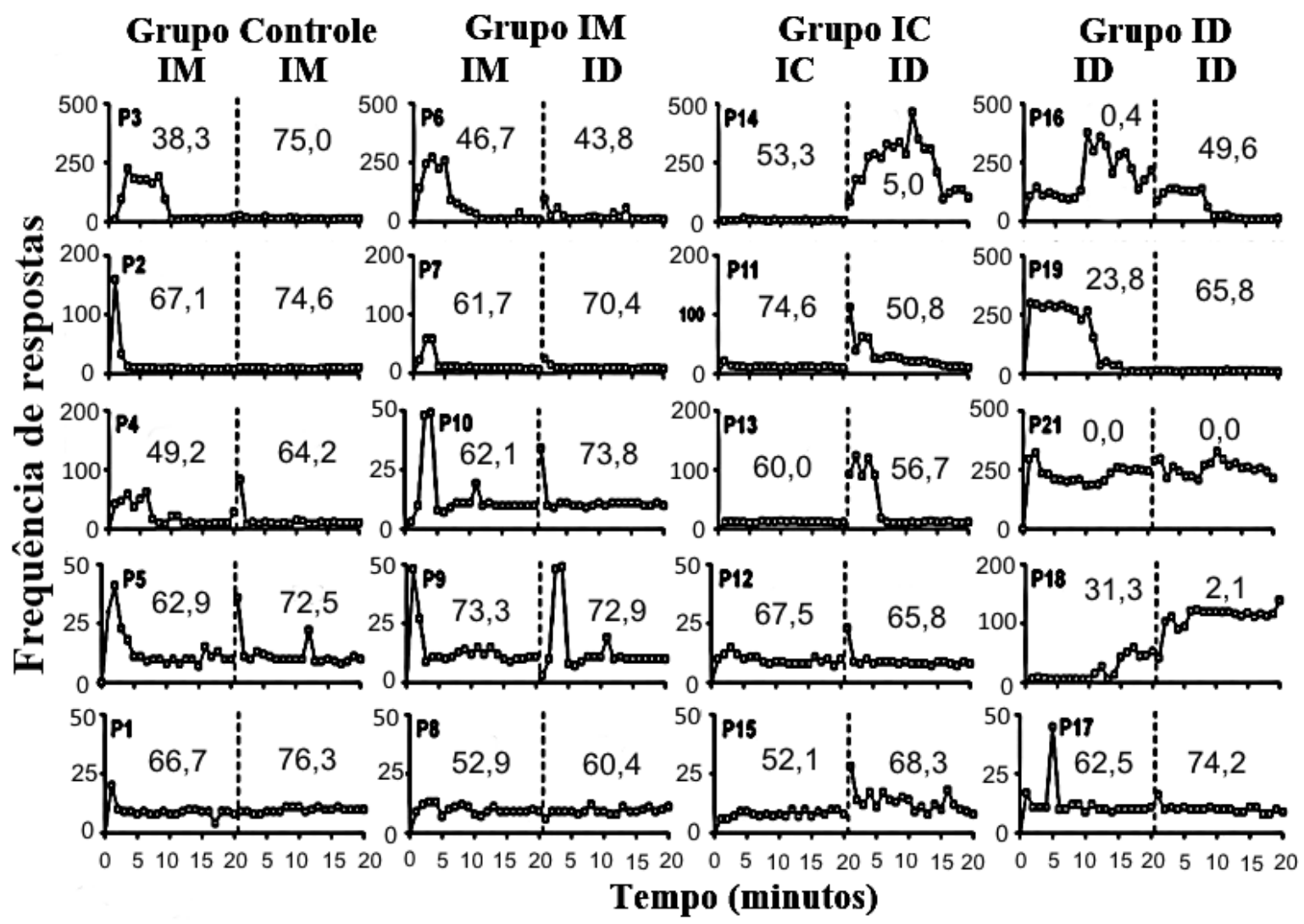

Figura 1. Respostas por minuto ao longo da Fase 1 e da Fase 2 dos participantes do Grupo Controle (primeira coluna da esquerda), Grupo IM (segunda coluna), Grupo IC (terceira coluna) e Grupo ID (última coluna da direita). A linha tracejada apresenta a mudança de fases. Os números representados na região central superior de cada fase representam a porcentagem de reforços obtidos. $O$ eixo $Y$ está com o máximo de 50 respostas para P1, P5, P8, P9, P10, P12, P15 e P17, 200 respostas para P2, P4, P7, P11, P13, P18 e 500 respostas para P3, P6, P14, P16, P19 e P21.

No início da Fase 1 (primeiros 5 minutos), para os participantes que receberam instrução mínima (Grupo Controle e Grupo IM), oito dos 10 participantes (P2, P3, P4, P5 P6, P7, P9 e $\mathrm{P} 10)$ emitiram taxas de respostas relativamente mais altas do que no restante da Fase 1. A média da taxa de respostas dos 5 minutos iniciais desses oito participantes ficou acima de $20 \mathrm{R} /$ min, enquanto $\mathrm{P} 1$ e $\mathrm{P} 8$ emitiram $11 \mathrm{R} / \mathrm{min}$. Três dos 10 participantes (P1, P2 e P9) obtiveram mais de $50 \%$ dos reforços disponíveis nos pri- meiros 5 minutos de sessão. Com a exposição continuada ao programa de reforço, as taxas de respostas tenderam a diminuir e a porcentagens de reforços obtidos tendeu a aumentar. Nos 5 minutos finais da Fase 1 todos os participantes dos Grupos Controle e IM emitiram $15 \mathrm{R} / \mathrm{min}$ ou menos e obtiveram mais de $50 \%$ dos reforços disponíveis neste período.

Os participantes do Grupo IC responderam em taxas relativamente mais baixas durante toda Fase 1 (entre 7 e $13 \mathrm{R} / \mathrm{min}$ ). Três dos cinco parti- 


\section{Tabela 2}

Média da Taxa de Respostas (Taxa Resp) e a Porcentagem de Reforços (Porc Refor) Obtidos dos 5 Minutos Iniciais (Min INI) e dos 5 Minutos Finais (Min FIM) de Cada Fase do Experimento e a Proporção de Mudança (Prop Mud) da Taxa de Respostas dos 5 Minutos Finais da Fase 2 em Relação aos 5 Minutos Finais da Fase 1

\begin{tabular}{|c|c|c|c|c|c|c|c|c|c|c|}
\hline \multirow[b]{3}{*}{ Grupo } & \multirow[b]{3}{*}{ Part } & \multicolumn{4}{|c|}{ Fase 1} & \multicolumn{4}{|c|}{ Fase 2} & \multirow[b]{3}{*}{$\begin{array}{l}\text { Prop } \\
\text { Mud }\end{array}$} \\
\hline & & \multicolumn{2}{|c|}{ Taxa Resp } & \multicolumn{2}{|c|}{ Porc Refor } & \multicolumn{2}{|c|}{ Taxa Resp } & \multicolumn{2}{|c|}{ Porc Refor } & \\
\hline & & $\begin{array}{l}\text { Min } \\
\text { INI }\end{array}$ & $\begin{array}{l}\text { Min } \\
\text { FIM }\end{array}$ & $\begin{array}{l}\text { Min } \\
\text { INI }\end{array}$ & $\begin{array}{l}\text { Min } \\
\text { FIM }\end{array}$ & $\begin{array}{l}\text { Min } \\
\text { INI }\end{array}$ & $\begin{array}{l}\text { Min } \\
\text { FIM }\end{array}$ & $\begin{array}{l}\text { Min } \\
\text { INI }\end{array}$ & $\begin{array}{l}\text { Min } \\
\text { FIM }\end{array}$ & \\
\hline \multirow{5}{*}{$\mathrm{CON}$} & $\mathrm{P} 1$ & 11 & 8 & 65 & 50 & 9 & 10 & 70 & 67 & 1,3 \\
\hline & $\mathrm{P} 2$ & 46 & 8 & 55 & 58 & 10 & 10 & 82 & 62 & 1,2 \\
\hline & P3 & 138 & 15 & 10 & 52 & 18 & 11 & 65 & 68 & 0,9 \\
\hline & $\mathrm{P} 4$ & 47 & 13 & 13 & 57 & 24 & 10 & 57 & 63 & 1,0 \\
\hline & P5 & 25 & 12 & 37 & 62 & 16 & 9 & 65 & 67 & 1,0 \\
\hline \multirow{5}{*}{ IM } & P6 & 228 & 15 & 10 & 60 & 44 & 11 & 23 & 55 & 1,7 \\
\hline & $\mathrm{P} 7$ & 32 & 8 & 32 & 52 & 13 & 9 & 65 & 60 & 1,2 \\
\hline & P8 & 11 & 9 & 42 & 55 & 8 & 10 & 55 & 55 & 1,0 \\
\hline & P9 & 21 & 10 & 62 & 65 & 24 & 10 & 63 & 62 & 1,4 \\
\hline & $\mathrm{P} 10$ & 24 & 10 & 32 & 68 & 15 & 10 & 68 & 67 & 1,2 \\
\hline \multirow{5}{*}{$\mathrm{IC}$} & $\mathrm{P} 11$ & 13 & 11 & 42 & 73 & 60 & 11 & 13 & 67 & 2,8 \\
\hline & $\mathrm{P} 12$ & 12 & 9 & 60 & 62 & 12 & 8 & 63 & 57 & 1,0 \\
\hline & $\mathrm{P} 13$ & 11 & 11 & 72 & 68 & 103 & 11 & 3 & 63 & 3,1 \\
\hline & $\mathrm{P} 14$ & 10 & 7 & 53 & 45 & 200 & 120 & 3 & 12 & 32,2 \\
\hline & P15 & 7 & 9 & 43 & 50 & 16 & 11 & 68 & 57 & 1,5 \\
\hline \multirow{5}{*}{ ID } & $\mathrm{P} 16$ & 119 & 208 & 0 & 2 & 123 & 10 & 8 & 65 & 0,3 \\
\hline & P17 & 19 & 10 & 50 & 60 & 12 & 9 & 70 & 58 & 1,0 \\
\hline & P18 & 7 & 51 & 45 & 0 & 89 & 119 & 8 & 0 & 2,2 \\
\hline & P19 & 288 & 10 & 0 & 62 & 11 & 10 & 63 & 57 & 1,1 \\
\hline & $\mathrm{P} 21$ & 259 & 250 & 0 & 0 & 262 & 246 & 0 & 0 & 1,0 \\
\hline
\end{tabular}

Nota. CON=Controle; IM=Instrução Mínima; ID=Instrução Discrepante; IC=Instrução Correspondente; Part=Participante; Taxa Resp=Taxa de respostas; Porc Refor=Porcentagem de reforços; Prop Mud=Proporção de mudança.

cipantes (P12, P13, P14) obtiveram mais de 50\% dos pontos disponíveis e P11 e P15 obtiveram 42 e $43 \%$, respectivamente, dos reforços disponíveis nos primeiros 5 minutos da Fase 1 . Nos últimos 5 minutos apenas P14 obteve menos de $50 \%$ dos reforços disponíveis (45\%).

No início da Fase 1 (primeiros 5 minutos), três dos cinco participantes do Grupo ID (P16,
P19 e P21) responderam em taxas superiores a $119 \mathrm{R} / \mathrm{min}$ e não obtiveram nenhum reforço; P18 emitiu, em média, $7 \mathrm{R} / \mathrm{min}$ e obteve $45 \%$ dos reforços disponíveis neste período e apenas P17 obteve 50\% dos reforços disponíveis e atingiu uma média de $19 \mathrm{R} /$ minutos nos 5 minutos iniciais. No final da Fase 1 (últimos 5 minutos) as taxas de respostas diminuíram para P17 e P19 
(média de $10 \mathrm{R} / \mathrm{min}$ para ambos), aumentaram para P18 (média de $51 \mathrm{R} / \mathrm{min}$ ) e permaneceram mais altas para P16 (média de $208 \mathrm{R} / \mathrm{min}$ ) e P21 (média de $250 \mathrm{R} / \mathrm{min}$ ). Dois dos cinco participantes (P17 e P19) obtiveram mais de 50\% dos reforços possíveis nos 5 minutos finais da Fase 1.

Resumidamente, o desempenho dos participantes do Grupo IC tendeu a ser mais eficaz na obtenção dos reforços disponíveis na Fase 1, uma vez que três dos cinco participantes obtiveram mais de $50 \%$ dos reforços disponíveis, contra três dos 10 participantes que receberam instrução mínima (Grupo Controle e IM) e um dos cinco participantes que receberam instrução discrepante. Estes resultados sugerem que a aquisição de um comportamento mais "eficaz" sob DRL (i.e., taxas de respostas relativamente mais baixas e porcentagens de reforços relativamente mais altas) foi facilitado pela instrução correspondente. No final da Fase 1, no geral, o desempenho dos participantes do Grupo Controle, IM e IC não foram muito diferentes em relação à capacidade de obter os reforços programados (i.e., todos obtiveram mais de $50 \%$ dos reforços disponíveis no período, exceto P10 que obteve $45 \%$ dos reforços disponíveis). Os participantes do Grupo ID, comparativamente, tiveram o pior desempenho em termos da eficácia na obtenção dos reforços disponíveis; apenas dois participantes atingiram mais de $50 \%$ dos reforços disponíveis na parte final da Fase 1.

Esses resultados, de modo geral, estão refletidos nas porcentagens de reforços obtidos durante toda a Fase 1 (valores à esquerda da linha tracejada na Figura 1). Considerando a eficácia em obter os reforços disponíveis na Fase 1 como um todo, o melhor desempenho foi o do Grupo IC em que todos obtiveram mais de $50 \%$ dos reforços disponíveis na Fase 1, seguido pelos participantes do Grupo IM e Controle (quatro dos cinco participantes no Grupo IM e três de cinco no Grupo Controle; mas P4 obteve 49,2\% dos reforços disponíveis na Fase 1), que obtiveram mais de $50 \%$ dos reforços disponíveis na Fase 1 e, por último, os do Grupo ID no qual apenas um participante (P17) obteve mais de $50 \%$ dos reforços disponíveis. Embora um desempenho "eficaz" possa ser facilitado pela instrução correspondente, um desempenho similar pode ser selecionado com a exposição continuada à contingência de reforço com o emprego de uma instrução mínima.

Com relação à Fase 2, na qual a instrução foi discrepante para todos os participantes, exceto para os do Grupo Controle que receberam a mesma instrução mínima já fornecida na Fase 1 , as taxas de respostas nos primeiros 5 minutos tenderam a ser maiores para os participantes dos Grupos IC e ID do que para os participantes dos Grupos Controle e IM. Três participantes do Grupo IC (P11, P13 e P15) e três do Grupo ID (P16, P18 e P21) atingiram uma média superior a $60 \mathrm{R} / \mathrm{min}$, no primeiro quarto da Fase 2 e obtiveram de 0 a $13 \%$ dos reforços disponíveis neste período. No mesmo período, os demais participantes do experimento (todos dos Grupos Controle e IM e dois de cada grupo IC e ID) tiveram médias inferiores a $24 \mathrm{R} / \mathrm{min}$ e obtiveram mais de $50 \%$ dos reforços disponíveis, com exceção de P6 (Grupo IM, média de 44 R/min e 23\% dos reforços). No final da Fase 2 (últimos 5 minutos do experimento), um participante do Grupo IC (P14, média de $120 \mathrm{R} / \mathrm{min}$ e $12 \%$ dos reforços disponíveis no período) e dois participantes do Grupo ID (P18, média de 119 R/min e P21, média de $246 \mathrm{R} / \mathrm{min}$, ambos com nenhum reforço obtido) mantiveram taxas de respostas relativamente altas; os demais participantes atingiram uma média igual ou inferior a $11 \mathrm{R} / \mathrm{min}$ e obtiveram mais de $50 \%$ dos reforços disponíveis.

Em síntese, os resultados da Fase 2 indicaram que, no geral, a maioria dos participantes do Grupo IC iniciou a Fase 2 com taxas de respostas relativamente elevadas (em comparação aos Grupos Controle e IM) e diminuíram a taxa de respostas ao longo da fase, enquanto que no Grupo ID, somente aqueles participantes que terminaram a Fase 1 emitindo baixas taxas de respostas (P17 e P19) iniciaram a Fase 2 com taxas de respostas relativamente baixas. Considerando a eficácia em obter os reforços disponíveis na Fase 2 como um todo (ver valores à direita da linha tracejada na Figura 1), o melhor desempenho foi o do Grupo Controle e IM (exceto P6, todos obtiveram mais de $60 \%$ dos pontos disponíveis na Fase 2), seguido pelos participantes do Grupo 
IC (quatro de cinco participantes obtiveram mais de $50 \%$ dos pontos disponíveis na Fase 2) e, por último os do Grupo ID no qual dois dos cinco participantes obtiveram mais de $50 \%$ dos reforços disponíveis.

A última coluna à direita da Tabela 2 exibe o cálculo da proporção de mudança das taxas de respostas. Valores próximos de 1 indicam menor proporção de mudança e cada 0,1 representa $10 \%$ de mudança, para mais ou para menos, na taxa de respostas do final da Fase 2 em relação ao final da Fase 1. Calixto et al. (2014) consideraram, baseados em Hayes, Brownstein, Haas et al. (1986), diferenças maiores que 50\%, para mais ou para menos, como significativas. Todos os participantes do Grupo Controle e IM emitiram taxas de respostas no final da Fase 2 muito semelhantes àquelas emitidas ao final da Fase 1. No Grupo IC, apenas o participante P14 mudou a taxa de respostas em mais de $50 \%$ e no Grupo ID dois participantes (P16 e P18) alteraram a taxa de respostas em mais de $50 \%$.

\section{Discussão}

O presente estudo investigou o efeito da história experimental com instruções mínimas, correspondentes ou discrepantes sobre o seguimento de uma instrução discrepante subsequente.

Os participantes que receberam a instrução correspondente (Grupo IC) tenderam a emitir, nos 5 minutos iniciais do experimento, taxas de respostas menores que os participantes dos demais grupos que receberam instrução mínima ou discrepante (exceto pelo desempenho de um participante de cada grupo: P1, P8 e P18, respectivamente) e, obtiveram uma maior porcentagem dos reforços disponíveis neste período. Estes resultados sugerem que a instrução correspondente facilitou o contato com as contingências programadas e, consequentemente, acelerou a aquisição do comportamento, corroborando os resultados de outras pesquisas da área (e.g., Athayde-Neto et al., 2015; Calixto et al., 2014; Hayes, Brownstein, Haas et al., 1986; Hayes, Brownstein, Zettle, Rosenfarb, \& Korn, 1986; Rosenfarb, Newland, Brannon, \& Howey, 1992).
Ao final da Fase 1, todos dos participantes que receberam instrução mínima ou correspondente emitiram taxas de respostas relativamente baixas e, desses, apenas um participante (P14) obteve menos de $50 \%$ dos reforços disponíveis. Dois dos cinco participantes que receberam instrução discrepante (P17 e P19) emitiram taxas de respostas relativamente baixas e obtiveram mais de $50 \%$ dos reforços disponíveis neste período. Adicionalmente, quatro dos cinco participantes do Grupo ID (P16, P18, P19 e P21) foram os que obtiveram as menores porcentagens dos reforços possíveis na Fase 1 como um todo (ver valores à esquerda da linha tracejada na Figura 1). Portanto, assim como no estudo de Calixto et al. (2014, Experimento 2), os participantes que receberam instruções mínimas e correspondentes diminuíram a taxa de respostas em menor tempo de sessão e garantiram o acesso a maior porcentagem de reforços programados.

O ponto a ser notado é que em Calixto et al. (2014, Experimento 1), uma resposta de consumação foi utilizada, como no presente estudo, e os participantes do Grupo IC emitiram taxas de respostas tão altas quanto aquelas dos participantes do Grupo ID. Os autores discutiram que a exigência da resposta de consumação pode ter tornado a instrução "pressione o botão de $5 \mathrm{em}$ 5 segundos" sob um DRL $5 \mathrm{~s}$ em uma instrução discrepante, uma vez que, pressionar o botão a cada 5 segundos não produzia o aumento no valor monetário obtido mas sim o aparecimento do smile e, sem a emissão da resposta de consumação, nada mais acontecia nos minutos subsequentes. No Experimento 2 a resposta de consumação foi removida e os resultados foram mais parecidos com os do presente estudo. $\mathrm{O}$ fato dos resultados do Grupo IC da presente pesquisa, que usou uma resposta de consumação, não terem sido parecidos com os descritos no Experimento 1 de Calixto et al. sugerem que, embora uma resposta de consumação possa gerar problemas com a correspondência entre uma instrução que descreva a taxa de respostas e a consequência (quando nada é dito sobre a resposta de consumação), esse nem sempre é o caso. Como a instrução, no presente estudo, não descrevia a 
resposta de consumação, essa topografia deve ter sido determinada não exclusivamente pelas instruções, mas também pelo contato com as consequências programadas (adição de $\mathrm{R} \$ 0,03$ ) no contador de reforços (cf. Albuquerque \& Paracampo, 2010).

No estudo de Calixto et al. (2014, Experimentos 1 e 2) todos os participantes que receberam instruções discrepantes na Fase 1 diminuíram a taxa de respostas e no presente estudo, dois participantes (P16 e P21) não diminuíram a taxa de respostas ao longo da Fase 1 (i.e., seguiram a instrução discrepante) sugerindo que, pelo menos em alguns casos, instruções podem ser seguidas mesmo quando as seguir não garante a obtenção de reforçadores programados (cf. Albuquerque et al. 2003; Paracampo, 1991; Ramos, Costa, Benvenuti, \& Andrade, 2015). Além disso, os resultados da Fase 1 dos participantes P16 e P21 do Grupo ID são similares aos resultados de Albuquerque et al. (2003, Experimento 1) em que quatro participantes, expostos à Condição D-C-D, seguiram a regra discrepante das contingências programadas nas duas fases em que elas foram fornecidas e apoiam a sugestão desses autores de que "o seguimento de regras discrepantes das contingências de reforço pode ser mantido independentemente de ser precedido por uma história experimental de reforço do seguimento de regra correspondente" (p. 105). É provável que o seguimento da regra discrepante, pelos participantes $\mathrm{P} 16$ e P21 do presente estudo, tenha sido mantido devido a uma história pré-experimental de seguimento de regras (cf. Albuquerque et al., 2003, Experimento 2).

O desempenho final na Fase 1 do presente estudo, foi semelhante entre os participantes que receberam instrução mínima e correspondente. Entretanto, não era possível saber se o comportamento dos que receberam instrução correspondente estava sob o controle da instrução, das contingências ou da interação entre ambos (cf. Albuquerque et al., 2008; Albuquerque \& Paracampo, 2010). Todavia, na Fase 2, quando ocorreu o fornecimento de novas instruções - discrepante para todos os grupos, exceto os participantes do Grupo Controle - observou-se diferença entre os participantes do Grupo IM e os do Grupo IC. No geral, os participantes do Grupo IM emitiram taxas de respostas mais baixas e obtiveram maiores porcentagens dos reforços disponíveis na Fase 2 do que os participantes com histórias de instrução correspondente (ver valores à direita da linha tracejada Figura 1). Esses resultados sugerem que, apesar de semelhantes no final da Fase 1, o comportamento dos participantes que receberam instrução correspondente provavelmente estava mais sob o controle das instruções discrepantes na Fase 2 do que o comportamento dos participantes do Grupo IM. Diversos outros estudos (e.g., Albuquerque et al., 2003; Athayde-Neto et al., 2015; Calixto et al., 2014; Hayes, Brownstein, Zettle et al., 1986; Joyce \& Chase, 1990; Podlesnik \& Chase, 2006; Rosenfarb et al., 1992; Shimoff et al., 1981), apontaram que o comportamento modelado pelas contingências (ou expostos diretamente às contingências com instrução mínima, como os do Grupo Controle e IM do presente estudo) foi mais sensível à mudança do que o comportamento instruído (como os do Grupo IC do presente estudo).

Os resultados obtidos na Fase 2 sugerem que houve diferença entre a história com instrução correspondente (Grupo IC) vs. instrução discrepante (Grupo ID) sobre a sensibilidade comportamental. Inicialmente, três participantes de cada grupo emitiram taxas de respostas relativamente altas (P11, P13 e P14; P16, P18 e P21) e nos 5 minutos finais da Fase 2, um participante do Grupo IC (P14) e dois do Grupo ID (P18 e P21) continuaram a seguir a instrução discrepante. Três dos cinco participantes do Grupo ID, mas apenas um do Grupo IC, obtiveram menos de $50 \%$ dos pontos possíveis na Fase 2 como um todo.

No estudo de Calixto et al. (2014, Experimento 2) os resultados indicaram que quando a contingência de reforço foi alterada e as instruções foram mantidas constantes, três de cinco participantes do Grupo IM, um participante do Grupo IC e três participantes do Grupo ID apresentaram alteração na taxa de respostas acima de $50 \%$, medido pela proporção de mudança. Os autores concluíram que os participantes que receberam a instrução discrepante na Fase 
1 alteraram mais a taxa de respostas na Fase 2 em comparação aos participantes que receberam a instrução correspondente. Assim como no estudo de Calixto et al., no presente estudo apenas um participante do Grupo IC alterou a taxa de respostas em mais de $50 \%$ contra dois participantes do Grupo ID. Esse resultado parece sugerir duas coisas: (a) História com regras correspondentes produzem mais resistência à mudança do que história com regras discrepantes (cf. Calixto et al., 2014; Martinez \& Tamayo, 2005) e (b) não houve diferença, no que diz respeito à avaliação da resistência do comportamento à mudança, entre o procedimento mais tradicional de manter a instrução e alterar as contingências de reforço (Calixto et al., 2014) e o procedimento de se alterar as instruções e manter constante a contingência de reforço (presente estudo) no que diz respeito à avaliação da resistência do comportamento à mudança.

$\mathrm{O}$ fato de que as taxas de respostas tenderam a ser relativamente altas e a porcentagem de reforço tendeu a ser relativamente baixa, nos primeiros 5 minutos da Fase 2, para seis dos 10 participantes dos Grupos IC e ID do que para quatro dos cinco participantes do Grupo IM (que receberam instruções discrepantes), sugere que as instruções, quer correspondentes ou discrepantes, diminuiu a sensibilidade do comportamento ao DRL em vigor, embora o resultado não tenha sido replicado para todos os participantes dos Grupos IC e ID.

Albuquerque e Silva (2006) e Albuquerque, Silva e Paracampo (2014) apontaram que o contato com a contingência em vigor em fases prévias do experimento aumenta a probabilidade do abandono de instruções discrepantes subsequentes. Isso foi verdadeiro para a maioria dos participantes da presente pesquisa. Entretanto, os resultados de P14 (Grupo IC) e P18 (Grupo ID) merecem uma avaliação mais detalhada. P14 do Grupo IC obteve mais de 50\% dos reforços possíveis na Fase 1, mas seguiu a instrução discrepante na Fase 2 obtendo apenas 5\% dos reforços possíveis. Especulativamente, pode-se dizer que o comportamento de P14 estava sob o controle da instrução correspondente no final da Fase 1 e os demais participantes estavam sob o contro- le tanto da instrução quanto da contingência de reforço e, por isso, abandonaram a instrução discrepante na Fase 2. Todavia, esta é uma análise post hoc e a história pré-experimental de seguir instrução (não avaliada no presente estudo) pode também ter contribuído para este resultado divergente.

P18 do Grupo ID emitiu baixas taxas de respostas até a primeira metade da Fase 1 (produzindo $45 \%$ dos reforçadores programados) e passou a emitir taxas de respostas relativamente mais altas, como especificado pela instrução discrepante na segunda metade da primeira fase (deixando de ganhar os reforçadores). Na Fase 2, P18 seguiu a instrução discrepante e obteve apenas $2 \%$ dos reforços possíveis para a Fase 2 como um todo. Para esse participante o contato com a contingência nos primeiros 5 minutos da Fase 1 não foi condição suficiente para o abandono do controle instrucional intra e entre as fases. Seguir instruções, mesmo após uma história de contato com as contingências foi observado em outros estudos (e.g., Albuquerque et al., 2003, Experimento 2; Shimoff et al., 1981). Não é possível explicar esse resultado a partir do procedimento adotado no presente estudo.

Os resultados do presente estudo estendem os achados dos estudos interessados em investigar os efeitos de diferentes instruções sobre a sensibilidade comportamental. Mais especificamente estende os resultados obtidos por Calixto et al. (2014) ao investigar, com um procedimento que mantinha constante o programa de reforço e alterava as instruções entre fases, o efeito da história experimental com regras mínimas, correspondentes ou discrepantes, sobre o seguimento de uma nova instrução discrepante. Em síntese, os resultados do presente estudo indicaram que instruções aumentam a probabilidade de um desempenho mais eficaz mais rapidamente na sessão (i.e., obtenção de maior quantidade de reforços possíveis com um menor número de respostas), mas que, expor os participantes a uma história com instrução correspondente com a contingência programada (ou discrepante, quando o comportamento não faz contato com a contingência programada) parece aumentar o tempo necessário para que os participantes aban- 
donem a instrução discrepante e façam contato com a contingência de reforço programada em uma fase teste posterior.. A exposição às contingências com instrução mínima aumenta a probabilidade de sensibilidade comportamental à contingência de reforço em vigor.

\section{Referências}

Albuquerque, L. C., de Souza, D. G., Matos, M. A., \& Paracampo, C. C. P. (2003). Análise dos efeitos de histórias experimentais sobre o seguimento subsequente de regras. Acta Comportamentalia, $11(1), 87-126$.

Albuquerque, L. C., dos Reis, A. A., \& Paracampo, C. C. P. (2008). Efeitos de histórias de reforço, curtas e prolongadas, sobre o seguimento de regras. Acta Comportamentalia, 16(3), 305-332.

Albuquerque, L. C., \& Paracampo, C. C. P. (2010). Análise do controle por regras. Psicologia USP, 21(2), 253-273.

Albuquerque, L. C., \& Silva, F. M (2006). Efeitos da exposição a mudanças nas contingências sobre o seguir regras. Psicologia: Teoria e Pesquisa, 22, 101-112. doi:10.1590/S010237722006000100013

Albuquerque, L. C., Silva, L. D. S., \& Paracampo, C. C. P. (2014). Análise de variáveis que podem interferir no comportamento de seguir regras discrepantes. Acta Comportamentalia 22, 51-71.

Athayde-Neto, C. A., Costa, C. E., \& Banaco, R. A. (2015). Efeitos da história comportamental e de instruções sobre a aquisição e a resistência à extinção em um esquema múltiplo FR DRL. Psicologia: Teoria e Pesquisa, 31(3), 365-374. doi:10.1590/0102-37722015032149365374

Calixto, F. C., Ponce, G. D., \& Costa, C. E. (2014.). O efeito de diferentes instruções sobre o comportamento em DRL e a sensibilidade comportamental. Acta Comportamentalia, 22, 201-217.

Catania, A. C. (1999). Aprendizagem: Comportamento, linguagem e cognição (D. G. de Souza, Trad.). Porto Alegre, RS: Artmed. (Original publicada em 1998)

Catania, A. C., Matthews, B. A., \& Shimoff, E. (1982). Instructed versus shaped human verbal behavior: Interactions with nonverbal responding. Journal of the Experimental Analysis of Behavior, 38(3), 233-248. doi:10.1901/jeab.1982.38-233
Cerutti, D. T. (1989). Discrimination theory of rule-governed behavior. Journal of the Experimental Analysis of Behavior, 51, 259-276.

Costa, C. E., \& Banaco, R. A. (2002). ProgRef v3: Sistema computadorizado para a coleta de dados sobre programas de reforço com humanos Recursos básicos. Revista Brasileira de Terapia Comportamental e Cognitiva, 4, 171-172.

Costa, C. E., \& Banaco, R. A. (2003). ProgRef v3: Sistema computadorizado para a coleta de dados sobre programas de reforço com humanos - Recursos adicionais. Revista Brasileira de Terapia Comportamental e Cognitiva, 5, 219-229.

Costa, C. E., Patsko, C. H., \& Becker, R. M. (2007). Desempenho em FI com humanos: Efeito da interação da resposta de consumação e do tipo de instrução. Interação em Psicologia, 11, 175186.

Galizio, M. (1979). Contingency-shaped and rule-governed behavior: Instructional control of human loss avoidance. Journal of the Experimental Analysis of Behavior, 31, 53-70.

Hayes, S. C., Brownstein, A. J., Haas, J. R., \& Greenway, D. E. (1986). Instructions, multiple schedules, and extinction: Distinguishing rule-governed from schedule-controlled behavior. Journal of the Experimental Analysis of Behavior, 46, 137-147.

Hayes, S. C., Brownstein, A. J., Zettle, R. D., Rosenfarb, I., \& Korn, Z. (1986). Rule- governed behavior and sensitivity to changing consequences of responding. Journal of the Experimental Analysis of Behavior, 45, 237-256.

Joyce, J. H., \& Chase, P. N. (1990). Effects of response variability on the sensitivity of rule-governed behavior. Journal of the Experimental Analysis of Behavior, 54(3), 251-262.

Lowe, C. F. (1979). Determinants of human operant behaviour. In M. D. Zeiler \& P. Harzem (Eds.), Reinforcement and the Organization of Behavior (pp. 159-192). New York: John, Wiley \& Sons.

Madden, G. J., Chase, P. N., \& Joyce, J. H. (1998). Making sense of sensitivity in human operant literature. The Behavior Analyst, 21, 1-12.

Martinez, H., \& Tamayo, R. (2005). Interactions of contingencies, instructional accuracy, and instructional history in conditional discrimination. The Psychological Record, 55, 633-646. 
Matos, M. A. (2001). Comportamento governado por regras. Revista Brasileira de Terapia Comportamental e Cognitiva, 3, 51-66.

Nevin, J. A. (1974). Response strength in multiple schedules. Journal of the Experimental Analysis of Behavior, 21(3), 389-408.

Paracampo, C. C. P. (1991). Alguns efeitos de estímulos antecedentes verbais e reforçamento programado no seguimento de regra. Psicologia: Teoria e Pesquisa, 7(2), 149-161.

Paracampo, C. C. P., \& Albuquerque, L. C. (2004). Análise do papel das conseqüências programadas no seguimento de regras. Interação em Psicologia, 8(2), 237-245.

Podlesnik, C. A., \& Chase, P. N. (2006). Sensitivity and strength: Effects of instruction on resistance to change. The Psychological Record, 56, 303-320.

Ramos, M. N., Costa, C. E., Benvenuti, M. F., \& Andrade, C. C. F. (2015). Efeito de regras inacuradas e monitoramento sobre desempenhos em programas de reforços. Psicologia: Reflexão e Crítica, 28(4), 813-822. doi:10.1590/16787153.201528420
Rosenfarb, I. S., Newland, M. C., Brannon, S. E., \& Howey, D. S. (1992). Effects of self-generated rules on the development of schedule-controlled behavior. Journal of the Experimental Analysis of Behavior, 58, 107-121.

Shimoff, E., Catania, A. C., \& Matthews, B. A. (1981). Uninstructed human responding: Sensitivity of low-rate performance to schedule contingencies. Journal of the Experimental Analysis of Behavior, 36, 207-220.

Skinner, B. F. (1969). Contingencies of Reinforcement: A theoretical analysis. New York: Appleton-Century-Crofts.
Recebido: 26/08/2015

$1^{a}$ revisão: 05/01/2016

Aceite final: 18/01/2016 